\title{
The Energy Endoscope: Real-time Detailed Energy Accounting for Wireless Sensor Nodes
}

\author{
Thanos Stathopoulos Dustin McIntire William J. Kaiser \\ Center for Embedded Networked Sensing \\ UCLA, Department of Electrical Engineering \\ \{thanos@cs.ucla.edu,dustin@seas.ucla.edu,kaiser@ee.ucla.edu\}
}

\begin{abstract}
This paper describes a new embedded networked sensor platform architecture that combines hardware and software tools providing detailed, fine-grained real-time energy usage information. We introduce the LEAP2 platform, a qualitative step forward over the previously developed LEAP [13] and other similar platforms. LEAP2 is based on a new low power ASIC system and generally applicable supporting architecture that provides unprecedented capabilities for directly observing energy usage of multiple subsystems in real-time. Real-time observation with microsecond-scale time resolution enables direct accounting of energy dissipation for each computing task as well as for each hardware subsystem. The new hardware architecture is exploited with our new software tools, etop and endoscope. A series of experimental investigations provide high-resolution power information in networking, storage, memory and processing for primary embedded networked sensing applications. Using results obtained in real-time we show that for a large class of wireless sensor network nodes, there exist several interdependencies in energy consumption between different subsystems. Through the use of our measurement tools we demonstrate that by carefully selecting the system operating points, energy savings of over $60 \%$ can be achieved while retaining system performance.
\end{abstract}

\section{Introduction}

Low energy operation continues as a fundamental limiting challenge for Wireless Sensor Network (WSN) systems. Most recently, the critical demand for high energy efficiency operation has increased yet further with the introduction of the new class of 32-bit processor-based WSN nodes that meet new application requirements for support of high performance sensors and complex algorithms $[13,11,10]$.
While these platforms present high peak operating power demand, prior work has shown that design objectives focusing on high energy efficiency along with proper scheduling of platform components yield low average energy operation [13]. However, as will be shown here, high efficiency may be achieved only if platform energy usage is measured at runtime, thereby revealing the effects of not only unpredictable application demands, but also of contention between platform components and computing tasks.

This paper describes a new hardware and software architecture that forms an Energy Endoscope with the first (to the best of our knowledge) integrated, low-power, real-time energy monitoring for WSN and other embedded systems. In contrast to prior systems that measure energy only at low temporal sample rates and determine the average over all platform components $[17,5,1,8]$, this new architecture resolves energy usage at real time and for each hardware subsystem, thus enabling observations both on a system prototyping as well as on a post-deployment level. The Energy Endoscope relies on a unique hardware and software architecture solution that includes a new Energy Management and Accounting (EMAP2) ASIC combined with operating system kernel features that nearly eliminate the prohibitively large energy and computing overhead that high rate energy accounting would levy on conventional platforms. Experimental results will demonstrate that energy optimization requires this capability and that it must be integrated with the platform in order to reveal the large amplitude energy excursions that can only be observed with high rate sampling at runtime. Further, as experimental results will show, only with this data can a system balance the conflicting demands of interdependent subsystems to avoid unnecessary energy usage. The first applications of the Energy Endoscope have immediately shown benefits for large energy optimization without loss in WSN node performance.

The Energy Endoscope enables energy optimizations that are adaptive to the many characteristics of applications 
which can only be known during runtime field operation. Applications now being developed with this new platform include seismic sensor networks, structural health monitoring networks, marine sensor systems, and biomedical monitoring. These important applications share increasingly common WSN requirements for support of high performance sensor systems, on-demand use of either long range or broadband wireless communication, and complex signal processing, sensor fusion, and networking algorithms.

The primary contribution of this paper is the introduction of a new platform architecture and a new set of hardware and software energy measurement tools, intended for both offline and online power and energy analysis. We utilize the unique capabilities of our hardware and software tools to provide unprecedented energy consumption visibility. We have used our tools for real-time profiling of several important subsystems such as CPU, memory, storage and networking. We show that there exist drastic differences in power dissipation over time depending on the choice of storage device and also reveal the significant effect of CPU power dissipation on storage and networking operations. We also profile a network file transfer operation between two LEAP2 nodes and discover the optimal CPU speeds for both the sender and the receiver, resulting in overall energy savings above $60 \%$.

\section{The LEAP2 Architecture}

In this section we provide an overview of current techniques for energy accounting and also describe the LEAP2 hardware architecture.

Traditional energy accounting techniques in WSNs as well as in mobile computing rely on external device support - such as oscilloscope sampling or data acquisition systems $[17,5]$ — or on internal device support such as "fuel gauge" circuits [1], or peripheral circuit modules [8]. Devices such as an oscilloscope provide high sampling rates and as such can acquire and display power dissipation data in real-time. However, they are external devices and may not probe all internal subsystem paths. Thus, they are impractical for use in deployed systems that need compact, low-power, real-time energy consumption information. A fuel gauge [1] or an integrated peripheral solution [8] is sufficiently low-power to be included in actual field applications; however, those devices cannot meet real-time constraints as they are limited either by their internal sampling rate or by the speed of the communications bus.

Power dissipation in previously developed systems is typically measured at the power supply or between the power supply connector and the node itself $[17,5,1,8]$. This enables system-wide power dissipation and energy consumption information. However, for the purposes of energy optimization, it is important to know the contribu-

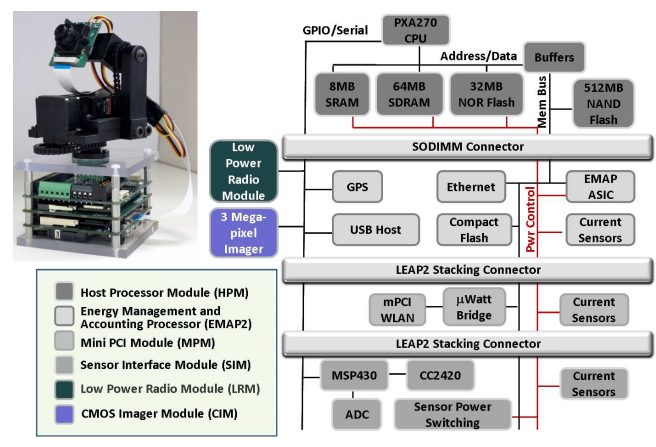

Figure 1. The LEAP2 hardware architecture.

tion of each subsystem - e.g CPU, memory or storage - to the total system energy consumption. With only singlechannel, combined energy information, resolving energy contributions associated with each subsystem may not be feasible in general due to uncertainties resulting from subsystem interdependence and resource contention. A typical approach [17] is to use microbenchmarks that exercise individual subsystems. By carefully reconstructing the event timeline, the system energy consumption can be attributed to the subsystem under investigation. This approach is therefore not real-time as post-facto processing is required. In addition component power dissipation is assumed to be constant throughout the duration of an operation [9]. This approximation may be adequate for very low-power components or very simple architectures. However, for highperformance WSN nodes where embedded processors may support dynamic voltage and frequency scaling, power draw depends on several factors such as CPU utilization, operating system support for on-demand frequency scaling, I/O intensity and others; therefore constant power dissipation cannot be safely assumed. Finally, we note that even though per-subsystem resolution could be achieved with an oscilloscope and a peripheral probe, in reality this solution would not be practical, in terms of scalability, ease of deployment and energy consumption for actual field deployments where energy dissipation measurements are most important.

\subsection{LEAP2 Platform Overview}

The second generation Low power, Energy Aware Processing (LEAP2) platform architecture overcomes these limitations and enables Energy Endoscope capability, providing an integrated, real-time detailed energy monitoring, capable of resolving energy usage at the level of each processing task and platform hardware component. This LEAP2 platform, shown in Figure 1 bears a similarity to LEAP and other previous energy aware architectures $[13,10,16]$ in that a high performance host processor and low power preprocessor are present. However, LEAP2 includes both a new Energy Management and Accounting 
Processor (EMAP2) ASIC device and new energy aware operating system kernel components. In contrast to previous platforms including LEAP, LEAP2 with the EMAP2 ASIC provides a qualitative and unprecedented advance in high accuracy, low overhead energy measurement of platform computing, storage, sensing, and communications devices at granularity levels previously unachievable.

At the foundation of the LEAP2 platform lies its energy management and accounting capabilities. On LEAP2 this feature is integrated into a dedicated ASIC, implemented in a micro-power antifuse-based field programmable gate array (FPGA). The FPGA has very low quiescent current - compared to SRAM-based FPGAs - of approximately $250 u \mathrm{~A}$. The EMAP2 ASIC, shown in Figure 2 performs continuous real-time energy monitoring, sophisticated power scheduling, and device resource multiplexing across the entire LEAP2 platform while requiring less than $6 \mathrm{~mW}$. Through the EMAP2 ASIC, LEAP2 peripherals may be scheduled for use only when needed and detailed energy information is gathered during their operation.

Energy usage information for individual platform subsystems including computational resources such as the PXA270 microprocessor, memory subsystems such as the SDRAM and SRAM, storage subsystems such as NOR flash and NAND flash, peripheral subsystems such as the Ethernet, 802.11, USB, Imaging, Compact Flash, and external sensors modules is available at millisecond accuracies. In addition, the EMAP2 ASIC energy data and scheduling controls are available to the host processor through a high bandwidth memory bus interface thereby minimizing measurement overhead issues. This enables the host processor to obtain energy usage information across a wide range of devices at millisecond intervals and with a minimal overhead. These features provide LEAP2 with a unique platform monitoring and control capability that can enable significant energy consumption optimizations. The following section provides details on the EMAP2 ASICs three critical functions on the LEAP2 platform: resource multiplexing, power scheduling, and energy accounting.

\subsection{The EMAP2 ASIC}

LEAP2 is an advance over previous architectures [13, $16,11,10]$ through inclusion of a Resource Multiplexor that enables an expanded set of peripheral and sensor interfaces and dynamic scheduling and energy measurement of power domains that may be added to or removed from the platform through an expandable energy management bus integrated into the LEAP2 stacking connectors.

Due to the large number of peripheral devices integrated into the LEAP2 platform and also expandable on the interboard stacking connector, a Resource Multiplexor was necessary. For point-to-point and point-to-multipoint buses such as UARTs, SPI ports and for traditional parallel mem-

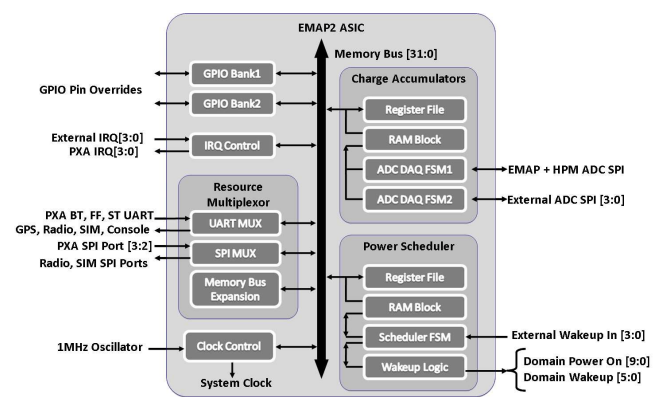

Figure 2. The EMAP2 ASIC.

ory buses this resource sharing feature is critical since the host processor is often insufficiently equipped with enough hardware resources to allow dedicated one-to-one connections. In the EMAP2 ASIC, serial bus multiplexor ports are implemented as a partially connected, switched network. Device ports are redundantly assigned to a subset of the host processor's available ports. This increases the probability of successfully mapping all active device ports to the host processor. A fully connected network was implemented, but found to require a substantial additional hardware investment over the partially connected implementation. In addition to the resource multiplexor, the EMAP2 ASIC provides a dynamic power scheduler capability (clocked by a configurable input signal driven from a micro-power silicon oscillator requiring only $70 u \mathrm{~A}$ at $3.3 \mathrm{~V}$ ), providing configurable scheduler resolution from 1 us to $10 \mathrm{~ms}$ based upon the configured input clock rate.

The EMAP2 ASIC design addresses the challenges arising from the competing requirements for microsecondresolution sampling and large data acquisition storage rate, low energy operation, and low processor computing overhead. The EMAP2 energy accounting module consists of a charge accumulation finite state machine (FSM) that acts as an energy integrator, two ADC data acquisition FSMs, and a large RAM block containing the charge accumulation data. The EMAP2 may address up to six Texas Instruments TLV2548 8-channel ADCs providing a total of 48 charge accumulation channels. Eight channels are allocated to the EMAP2 module's peripherals such as Ethernet, USB, quick capture camera, and compact flash. Eight additional channels are allocated for the host processor module (HPM) PXA270 core, SDRAM, NAND and NOR flash, and SRAM. The EMAP2 and HPM accumulation channels are provided by the first of the two data acquisition modules and interface the ADCs over SPI port1. The remaining 32 channels are controlled via the second data acquisition module which can interface up to four additional ADCs over SPI port2. The parallel SPI acquisition channels reduce acquisition latency and required SPI bus clock frequency. The host processor interfaces with the energy accounting mod- 
ule through a set of configuration registers and the charge accumulation data is read directly from the RAM block. Host reads from the charge accumulation RAM is arbitrated by the energy accounting module with priority given to the host processor reads. Write from the ADC data acquisition FSMs may be delayed by the memory arbiter during host processor access. The current sensor sample period is configurable through a 24-bit configuration register providing ADC sample rates from $25 \mathrm{KHz}$ to less than $0.25 \mathrm{~Hz}$. All SPI bus data samples transfers are pipelined such that commands for sequential channels conversions are interleaved with the readback of the current channel data to minimize latency. Readback from the last active channel on a given $\mathrm{ADC}$ causes the ADC powerdown command to be issued, reducing $\mathrm{ADC}$ idle power.

The EMAP2 architecture is also designed to reduce processor overhead associated with energy accounting. The host processor reads charge accumulation and accumulation count information directly from the accumulation RAM block in 32-bit read cycles. Since the EMAP2 is able to provide accumulation RAM block access in typically less than $40 \mathrm{~ns}$, data readback is extremely low overhead. The charge accumulation data is aliased to two separate host processor memory address windows providing a rapid, incremental charge reading capability necessary for low overhead energy accounting. When all of the 48 EMAP2 channels are enabled and accumulating, host processor readback of accumulation data and count will require only 192 bus cycles (taking less than 8us) assuming no bus arbitration loss. Even when using $1 \mathrm{~ms}$ readback periods, the host processors memory access overhead is then less than $0.8 \%$.

\section{Energy Measurement Software Tools}

Using the capabilities of the EMAP2 ASIC, our goal is to provide a set of software tools that can be used for rapid real-time energy observation as well as for detailed energy analysis. with a low-power, wireless, mobile computing platform therefore developed two energy measurement software tools: Etop, a user-space energy consumption observation tool and endoscope, a low-overhead kernel-space energy measurement tool.

\section{$3.1 \quad$ Etop}

Etop is a user-space tool that enables rapid observation of energy consumption, when running an arbitrary set of processes. Using etop, an application developer can easily visualize - in real-time - the power draw of subsystems and energy consumption of running processes. Therefore, etop is primarily intended as a pre-deployment observation tool Nevertheless, due to its rapid real-time information display, it can also be used as a runtime debugging tool,

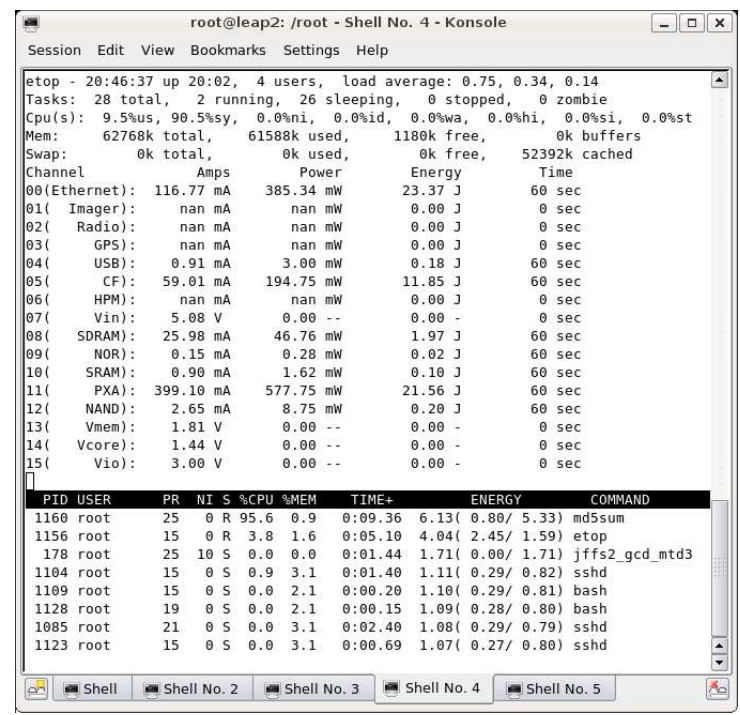

Figure 3. Etop screenshot displaying per-subsystem power and energy information as well as per-process energy consumption.

for quickly ascertaining system and process energy performance, as well as for understanding the energy behavior of processes in a dynamic environment.

Etop is based on the well-known UNIX program top. Etop adds two additional capabilities: per-subsystem current, power and energy information and per-process energy accounting. Figure 3 presents a screenshot of etop, with the top part displaying per-subsystem information and the bottom part displaying per-process energy consumption.

Etop's per-subsystem information is directly linked to the output of the EMAP2. On every refresh cycle (a usercontrolled parameter that top provides, with a default of 3 $\mathrm{sec}$ ), etop presents current, power and energy consumption information of individual subsystems. It also provides voltage information for the four voltage-only channels. Current and power values are by default averaged over the refresh period; maximum values can also be displayed.

To provide etop with per-process energy accounting, we introduced a modification to the Linux OS scheduler so as to record energy consumption information, in a manner similar to ECOsystem [18]. Specifically, we augmented the task_struct structure (that contains information about a specific process) with two energy containers: one for user-level information and one for kernel-level information. On every scheduler tick, Linux determines whether the current process time slice executed in user or kernel mode and charges an appropriate container accordingly. This information is used by Linux (and top) to determine the fraction of CPU time spent in kernel (system) mode and in user mode. We read the EMAP2 charge values from all channels on every 
scheduler tick and charge the corresponding user- or kernellevel process energy container. As a result, we can disambiguate between energy consumption that occurred when a process was performing work in support of a user mode task and when the system was performing work during the process time slice in support of kernel mode tasks. Both these values are exported using the /proc interface, in a manner similar to $/$ proc/ $<$ pid $>/$ stat. Etop then reads those values and displays them in standard top-like column format.

This development of etop provides both an immediately valuable and also new online profiling capability. However, it is important to note that additional opportunities for extensions lie ahead. Specifically, etop attributes energy consumption in a synchronous manner. In the future, asynchronous operations, i.e., operations that do not necessarily occur when the process that caused them is running may be included. Typical examples of those operations include networking and disk I/O. On the other hand, one can assume that when a process is running, it effectively has control of the CPU and memory. As a result, when calculating the energy consumption of a process, we only factor in the PXA (CPU) channel and the SDRAM (memory) channel. An important extension of etop addressing the problem of accounting for asynchronous operations will be to associate the initiating process identifier with a particular operation (i.e., disk I/O or networking I/O) and then retroactively charge the appropriate process. We thus plan to develop this extended per-process energy accounting in the future, utilizing a method similar to Resource Containers [2].

\subsection{Endoscope}

Since etop displays system energy performance in realtime it is not optimized for in-depth detailed measurements. Etop is a userspace process with non-trivial memory and CPU usage (especially at refresh rates higher than $1 \mathrm{~Hz}$ ) and as such can interfere with the measurements themselves.

To provide high-rate sampling in software with a very low energy overhead, we implemented the endoscope kernel module. Endoscope can read the EMAP2 registers at frequencies as high as the default Linux scheduler tick resolution (100 Hz in our system) and with very low CPU overhead, as no expensive user-kernel boundary crossings are required. The results are then stored in a circular buffer in kernel memory - a very fast and low-overhead operation. We utilize a standard/proc interface for userspace data display and control purposes. To avoid frequent periodic polling of the /proc interface from userspace, the circular buffer has sufficient memory to store several minutes' worth of real-time data - up to 2 minutes of continuous sampling of 16 channels at $100 \mathrm{~Hz}$ in our current implementation. To avoid buffer overrun, a userspace application only needs to read from the /proc interface at an interval that is slightly smaller than the buffer's capacity at a specific sampling rate.

\begin{tabular}{|c|cc|}
\hline & Etop & Endoscope \\
\hline Execution space & User & Kernel \\
Kernel modifications & Scheduler for & Kernel Module \\
& per-process data & \\
Data display interface & top-like & /proc \\
CPU usage & $1-25 \%$ & Negligible \\
Sample storage & No & Yes \\
Real-time data collection & Yes & Yes \\
Real-time data display & Yes & No \\
Per-subsystem resolution & Yes & Yes \\
Per-process resolution & Yes & No \\
\hline
\end{tabular}

Table 1. Qualitative comparison of Etop and Endoscope

Endoscope's negligible operating overhead, combined with the minimal power requirements of the EMAP2 ASIC, have enabled post-deployment monitoring and analysis of the power behavior of applications, both on a single node and on the entire network. As a result, we used endoscope extensively to collect our experimental dataset. Endoscope, unlike etop, measures energy consumption of entire subsystems rather than that associated with individual processes. As a result, when conducting our experiments, care is taken to ensure that endoscope measures energy solely attributed to a single application. For this development of endoscope, this procedure is effective for WSN systems that typically support one dominant application, or a set of applications that can be considered dominant. At the same time, as for etop, extensions to endoscope will enable increasingly complex WSN computing application support. Table 1 summarizes the capabilities and differences of our software tools.

\section{Single-node Energy Profiling}

In this section we focus on profiling the energy consumption of a single LEAP2 node. Our experiments aim to showcase the energy consumption of critical subsystems, such as CPU, memory, storage and networking. We also outline potential optimizations that can yield better energy efficiency.

Throughout our experiments, we utilized three metrics: instantaneous power dissipation over time, total energy consumption and the energy-latency product. Instantaneous power dissipation over time captures variations in power dissipation and is especially important in cases where power dissipation is not uniformly distributed in time. We calculate power dissipation by converting charge values from the EMAP2 into instantaneous current dissipation and then multiplying with the corresponding voltage channel measurement. The energy metric is useful when optimizing energy consumption for the entire system or for a specific set of subsystems. We calculate energy consumption by integrating instantaneous power values over a specific time period. The energy-latency product [6] is useful when op- 


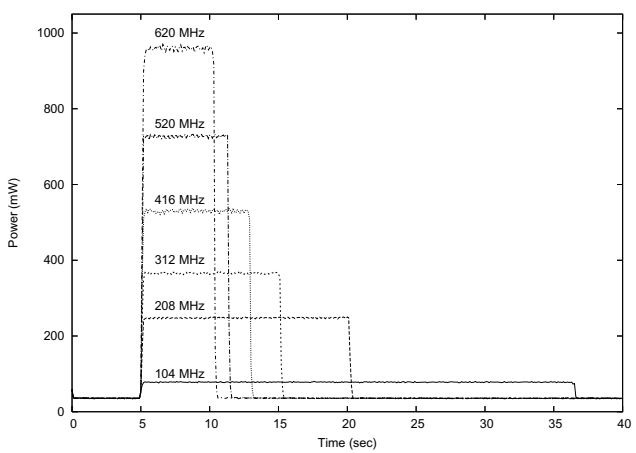

Figure 4. CPU power dissipation over time, when running dcache_test, for six CPU speeds.

timizing for energy consumption as well as latency. Several WSN applications tend to be latency-tolerant; therefore trading off increased latency for lower energy consumption is acceptable and in that case the energy consumption metric is more useful. However in cases where specific latency bounds need to be met, or when optimizing for both energy consumption and performance the energy-latency product is the more appropriate metric. Ideally, the desired operating point of a system or application is the one that minimizes both energy consumption and the energy-latency product.

Our experimental data was collected in real-time, by using endoscope with a sampling rate of $10 \mathrm{~Hz}$. Each experiment was repeated 5 times. We report averages when presenting energy (Joules) and energy-latency product (Joules*sec) values and use instantaneous values from a single experiment when presenting power values.

\subsection{CPU Subsystem Energy Profile}

The CPU is arguably the most important component of a platform, as every other subsystem depends on it, either directly or indirectly. It also is often the largest power consumer; therefore it needs to be energy efficient. We thus focus on investigating the power dissipation and energy consumption of the PXA270 CPU used in the LEAP2 node. Prior work $[13,1,9]$ has demonstrated that a more capable CPU (such as the PXA255 or PXA270), while incurring significantly higher peak power dissipation than an MCU (e.g., MSP430) is in fact more energy efficient while under load, due to its architectural advantages - higher clock speed, L1 caches, hardware MMU, multi-stage pipeline etc. Therefore, our goal for this set of experiments was to verify whether a CPU running at a higher speed is still more energy efficient than the same CPU running at a lower speed.

Since we wanted to measure only the CPU power dissipation, we required a test application that would not considerably utilize any other system resources, such as stor-

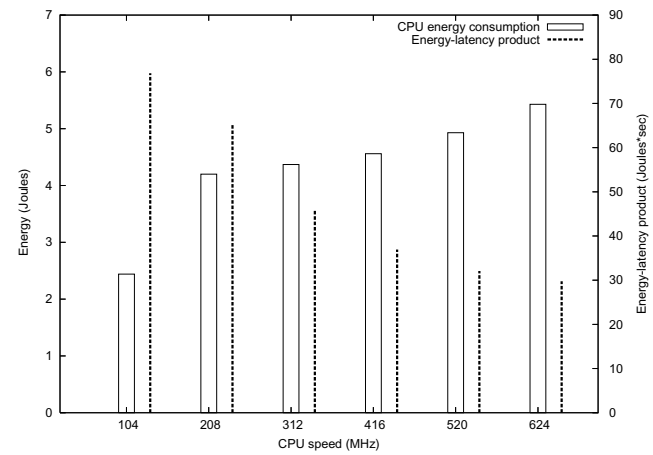

Figure 5. CPU energy (left) and energy-latency product (right) when running dcache_test, for six CPU speeds.

age or even RAM. We therefore used a simple test program called dcache_test, that computes the sum of an array of integers. The array's total memory size is 4 Kbytes so that it fits into the processor's data cache. The computation is continuously repeated, up to a given number of consecutive runs. Therefore, the CPU utilization during our test program's execution is very close to $100 \%$, while all other subsystems including SDRAM are not utilized.

We utilized the ondemand Linux CPU governor to dynamically change the CPU speed. This particular CPU governor automatically adjusts the CPU speed based on the observed CPU utilization. Under default settings, this governor increases the CPU speed to the maximum allowed value when the idle time is less than $20 \%$. It then tries to find the lowest allowed CPU speed that can sustain the load while keeping the idle time over $30 \%$. For each set of experiments, we gradually increased the maximum allowed CPU speed, from $104 \mathrm{MHz}$ all the way to $624 \mathrm{MHz}$.

Figure 4 presents the CPU power dissipation over time when running dcache_test, for the six different PXA CPU speeds. To indicate the difference in power dissipation between the idle CPU state and the loaded CPU state, we started our test program five seconds after endoscope. As can be seen in Figure 4, the CPU power dissipation raises considerably at $t=5 \mathrm{sec}$. Moreover, there is almost an order-of-magnitude difference between the power dissipation of the lowest CPU speed setting $(80 \mathrm{~mW})$ and the highest one $(970 \mathrm{~mW})$. This difference is even more apparent when comparing the idle CPU power dissipation at $104 \mathrm{MHz}$ with the loaded CPU power dissipation at 624 MHz. Figure 4 also indicates that there is a non-linear increase in the CPU power dissipation as the CPU speed increases. This is due to dynamic voltage and frequency scaling (DVFS) where changes in CPU frequency are accompanied with changes in core voltage. Since power is the product of current and voltage, the increase in power dissipation for different frequencies is expected to be non- 

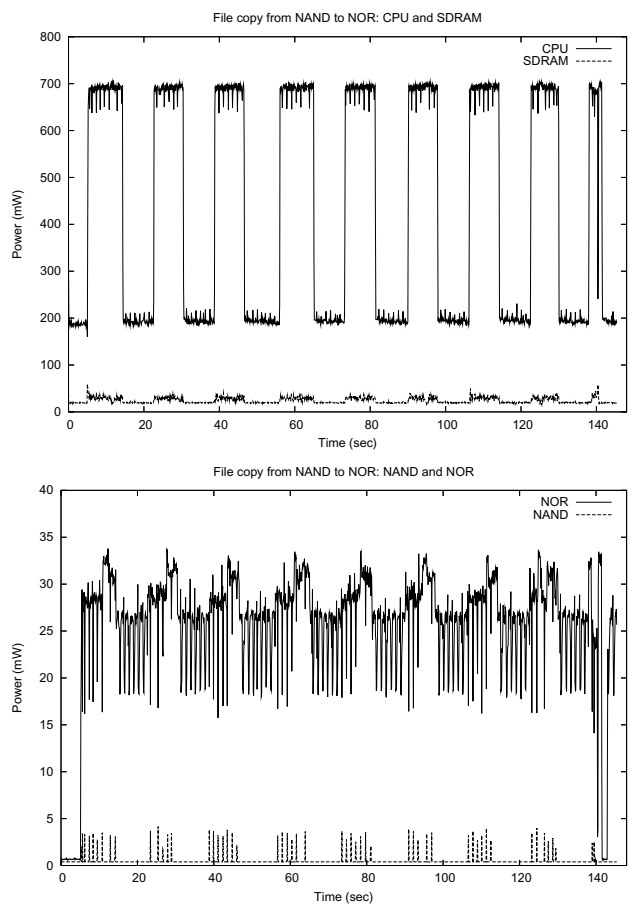

Figure 6. CPU, SDRAM, NAND and NOR power draw over time when copying a $7 \mathrm{Mb}$ file from NAND to NOR.

linear. As endoscope and EMAP2 can sample both the CPU current draw and the Vcore voltage at the same time, CPU power dissipation can be accurately estimated for each sample, thus capturing the non-linear DVFS effect.

Even though a lower CPU speed results in a considerable increase in completion time, the actual CPU energy consumption is lower, as Figure 5 shows. The increase in completion time at lower speeds is not significant enough to offset the drastic decrease in peak power draw, We therefore conclude that unlike the case where a faster 32-bit CPU is more energy efficient than an MCU despite the higher peak power dissipation [13], when comparing different speeds of the same DVFS-enabled CPU, the lowest CPU speed requires the least amount of energy for the same workload. The above result however does not consider latency: even though the lowest CPU speed draws the least amount of energy, it requires the most amount of time to complete. If instead we use the energy-latency product, the highest CPU speed becomes the most energy-latency efficient point.

Our results above contain information about a single subsystem; the CPU. A valid question then is whether results still hold when taking into account all subsystems that have a considerable energy consumption, such as network interfaces and SDRAM. We can assume that for applications that perform computation without need for communication (e.g., signal processing), networking subsystems can be in a
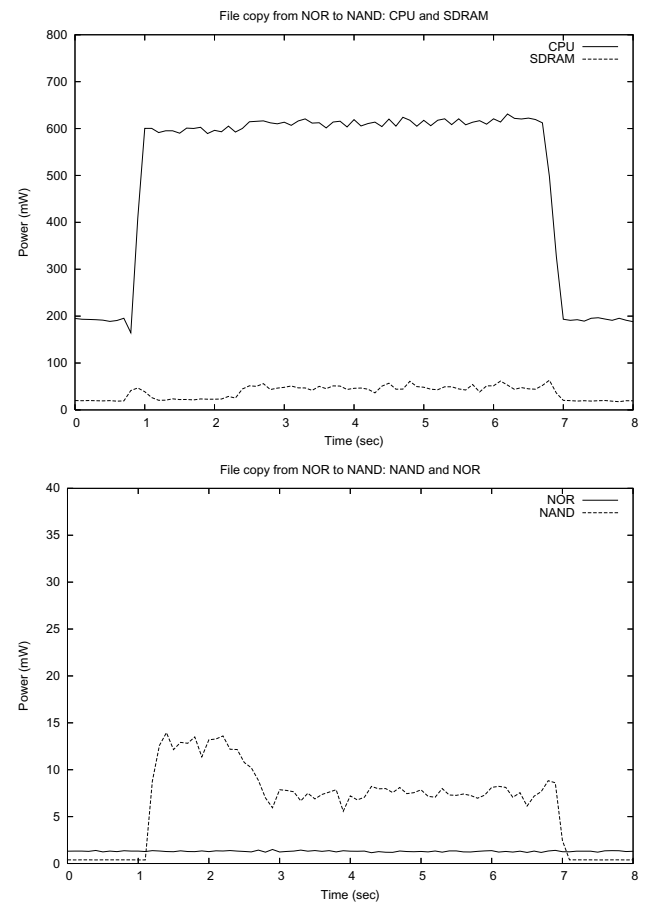

Figure 7. CPU, SDRAM, NAND and NOR power draw over time when copying a $7 \mathrm{Mb}$ file from NOR to NAND.

powerdown state. Therefore, a longer execution time would not affect the energy consumption of those subsystems. On the other hand, even though SDRAM may or may not be used in a CPU-intensive application, it cannot be turned off at runtime, without significantly modifying the operating system itself. SDRAM peak power draw is at least an order of magnitude lower than that of the CPU; consequently, even when taking SDRAM into account, the lowest CPU speed would still be the most energy efficient, even though the difference between it and the highest CPU speed would be slightly smaller. However, as will be shown in following sections, in applications that utilize several subsystems the lowest CPU speed is no longer the most energy efficient.

\subsection{Storage Subsystem Energy Profile}

Our next set of experiments focuses on the storage subsystem - an important component for both WSN nodes and embedded systems in general. Our goal for this set of experiments is two-fold. First, we want to investigate the differences in power dissipation between the two types of non-volatile storage included on LEAP2: NOR flash and NAND flash. Those two flash types differ considerably in their operational characteristics, so we expect significant differences in their power profiles as well. Second, we want to investigate the interdependencies between the stor- 
age subsystem and the computation subsystem (CPU and SDRAM). Even though similar tradeoffs have been studied in embedded systems [7], those studies were structured around offline analysis (based on data sheets) while our work focuses on real-time experimental validation. Our experimental setup consisted of copying a $7 \mathrm{MB}$ file from one storage subsystem to the other (i.e., from NAND to NOR or from NOR to NAND), with the CPU operating at a constant speed of $520 \mathrm{MHz}$. Linux VM file caches were flushed after each experiment to avoid faster completion time due to caching. The subsystems measured with endoscope for this set of experiments were CPU, SDRAM, NAND and NOR.

Figure 6 shows the power dissipation of the four subsystems over time for a file copy from NAND to NOR, while Figure 7 depicts the results for the file copy from NOR to NAND. These figures indicate very significant differences between the two flashes. First, copying to NOR takes approximately 140 seconds, while copying to NAND only takes approximately 6 seconds. Second, NOR peak power when writing and erasing is more than twice that of the equivalent NAND peak power. On the other hand, NAND reads are more expensive, power-wise than NOR reads: in fact, NOR reads don't cause any discernable difference above NOR's idle power dissipation. These results are in accordance with the characteristics of NOR and NAND flashes. NAND flashes are optimized for sequential block access and have much faster write and erase speeds than NOR flashes, therefore the write operations on NAND execute much faster and with considerably less power than on NOR. Conversely, NOR flashes support random-access reads (unlike NAND flashes), therefore read operations on NORs execute faster and require significantly less power.

NOR energy profile: The most important difference however exists in the power variations over time for NOR versus NAND. For NOR, CPU power dissipation over time has a pulse-like shape, while for NAND, CPU power dissipation is almost constant. The differences are due to the way that each flash type (and its associated drivers) handles write and erase operations. The NOR driver (Memory Technology Device-MTD) and filesystem (Journaling Flash File System-JFFS2) implement erase suspend on write. Therefore, write and erase operations in NOR flash are serialized: during a write operation, an erase operation cannot proceed, and vice-versa. This behavior explains the pulse-like shape of Figure 6. Write operations to NOR happen when CPU power peaks: this is corroborated by the fact that peaks in the SDRAM and NAND subsystems are correlated with peaks in the CPU subsystem, indicating sequences of reads and writes. When CPU power drops-indicating that the CPU is idling - so does NAND and SDRAM power dissipation: however, NOR power dissipation remains high, since during that time, the NOR flash is erasing blocks that will be needed for the next write operation. This sequence of

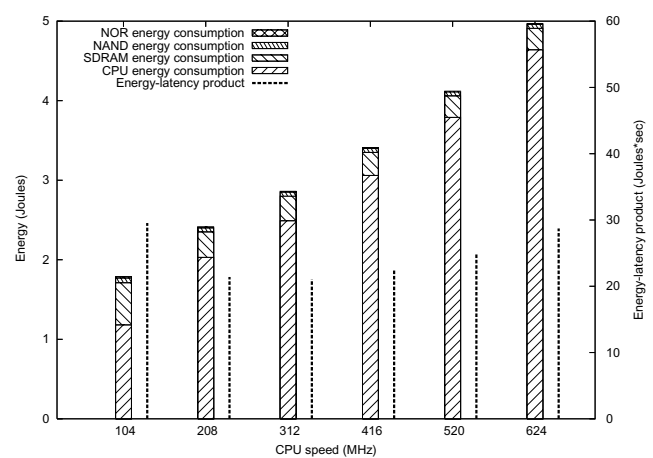

Figure 8. Average CPU, SDRAM, NOR and NAND energy (left) and energy-latency product (right) when copying a 7MB file from NOR to NAND, for six CPU speeds.

events was verified using the Linux Trace Toolkit [3].

NAND energy profile: When writing from NOR to NAND, we observe that during the first 1.3 seconds of the file copy, NAND power is at its peak. At the same time, CPU and SDRAM have not reached their peak power values; this is more evident in SDRAM. After this initial time period, CPU and SDRAM power dissipation reach peak values while NAND power dissipation decreases.

Using LTT we observed that during this initial time period, the YAFFS2 filesystem (used for NAND) executes a series of erase operations. Those operations do not continue throughout the entire file copy duration. During a copy to NAND, YAFFS2 determines how many blocks (if any) need to be erased and proceeds to erase those blocks in parallel with normal write operations. Since the file size is considerably smaller than the total NAND capacity, and considering that YAFFS2 implements wear-leveling in software, the erase blocks are significantly fewer than the total blocks that need to be written. NAND power dissipation peaks as blocks are being erased and written in very short succession. After the erase operations cease, writes can proceed at full speed, therefore the CPU and SDRAM subsystems are more heavily utilized and their power dissipation peaks.

SDRAM contribution: Regardless of whether we write to NOR or NAND, we notice that SDRAM power dissipation raises above idle during write operations. Even though both YAFFS2 and JFFS2 keep several data structures in SDRAM, their memory footprint is not large enough to explain the significant rise in SDRAM power dissipation. Instead, SDRAM is being used extensively because the Linux Virtual File System (VFS) implements memory caching in order to improve read and write access to files. Files are first written to SDRAM - or more accurately to the page cache - before they are committed to storage.

Effects of CPU speed: Caching is the first reason why $\mathrm{CPU}$ power dissipation is high during writes, as the $\mathrm{CPU}$ is 
also involved in this operation. The second one is software error correction. Neither the NOR or NAND modules in LEAP2 include hardware-supported error correction checks (ECC); therefore the filesystem needs to implement ECC in software. ECC is critical to the correct operation of NAND flashes, as those flashes always contain bad blocks. ${ }^{1}$

The aforementioned reasons indicate a significant dependency between CPU speed and write performance. Figure 8 shows a per-component energy cost as well as the energylatency product for the file copy from NOR to NAND, for six CPU speeds. Again, the lowest CPU speed results in the minimal energy consumption. The energy-latency product however is minimized when the CPU is operating at 312 $\mathrm{MHz}$, with $208 \mathrm{MHz}$ also being very close to the minimum. In Section 4.1, the test application was exclusively CPU-bound. Operating at the highest CPU speed resulted in a factor of five reduction in completion time, while energy consumption was increased by less than a factor of three; hence the energy-latency product was minimized at the highest CPU speed. The file-copy operation however is not exclusively CPU-bound as it involves $\mathrm{I} / \mathrm{O}$. As a result, in higher speeds, the CPU is not fully utilized. The peak power draw of the CPU operating at $520 \mathrm{MHz}$ under $100 \%$ load is around $735 \mathrm{~mW}$ (Figure 4), while the peak power dissipation in Figure 7 is around $600 \mathrm{~mW}$-hence the CPU load is not at $100 \%$. Therefore, lower CPU speeds yield similar performance benefits while requiring less energy due to lower idle and peak power dissipation. Deviating from the minimum energy-latency product thus leads to "diminishing returns", in terms of energy at speeds lower than the optimal and in latency in speeds higher than the optimal.

One final observation regarding the effect of CPU speed is its contribution to energy consumption versus that of the storage device. Figure 8 indicates that the CPU contribution ranges from $65 \%$ to $93 \%$ while the NAND contribution ranges from $3.3 \%$ to $1 \%$. This result is in sharp contrast with mote-class systems, where the CPU energy consumption and the storage (NAND or NOR) energy consumption are within the same order of magnitude $[12,4]$.

\section{Multi-Node Energy Profiling: Node-to- Node File Transfer}

In Section 4, we focused on profiling the energy consumption of various subsystems on a single LEAP2 node. In this section we aim to profile the energy consumption of an application that exercises multiple subsystems in multiple nodes. A network file transfer application satisfies our requirements, as it involves multiple nodes by definition and also exercises some of the most important subsystems on a

\footnotetext{
${ }^{1}$ Manufacturers ship NAND flashes with some bad blocks to keep their yield high and the cost low.
}

\begin{tabular}{|c|cc|cc|}
\hline & \multicolumn{3}{|c|}{$\begin{array}{c}\text { Energy Consumption (Joules) } \\
\text { Min } \\
\text { Value }\end{array}$} \\
& \multicolumn{2}{|c|}{$\begin{array}{c}\text { Max Speed } \\
\text { (Snd/Rcv) }\end{array}$} & $\begin{array}{c}\text { Value } \\
\text { CPU Speed } \\
\text { (Snd/Rcv) }\end{array}$ \\
\hline Sender & 26.12 & 208524 & 58.87 & 104624 \\
Receiver & 30.67 & 208208 & 64.21 & 104624 \\
Combined & 57.35 & 208208 & 95.95 & 104624 \\
\hline
\end{tabular}

Table 2. Minimum and maximum sender, receiver and combined energy consumption and sender - receiver CPU speed during a $20 \mathrm{MB}$ file transfer over $802.11 \mathrm{~b}$.

node. In addition, it allows us to demonstrate the online profiling capabilities of Endoscope, on a network level.

Our experimental setup consisted of two LEAP2 nodes - one sender and one receiver - with their wireless interfaces set to ad-hoc mode. In each experiment, the receiver would initiate a $20 \mathrm{MB}$ file transfer, using TCP. The location of the file in the sender as well as the file's destination on the receiver was the NAND flash. Endoscope was used on both the sender and the receiver to collect experimental data. In order to synchronize the timelines of the sender and the receiver for the purposes of data processing, both nodes were running NTP.

From Section 4.2 we saw that the CPU speed has a significant impact in energy consumption when writing to stable storage. Therefore, we expect the receiver's energy consumption to depend on the CPU speed. Furthermore, on the sender side, a lower CPU speed can potentially result in increased latency, as the CPU might not be able to fill the network card's transmit buffers fast enough. Results from Section 4.1 indicated that a lower CPU speed can be more energy efficient, even though latency is increased. However, in the network file transfer case, the considerable energy consumption of the wireless interface needs to be taken into account. A reduction in latency could result in further energy savings as the networking subsystem could be placed into a low-power state (or even powered down) after the file has been transferred. In order to verify our expectations we therefore conducted experiments with various CPU speeds, for both the sender and the receiver.

During the course of our experiments, all available frequency values were explored other than at $416 \mathrm{MHz}$. It was discovered that the pcmcia driver for the wireless interface would not function correctly at this frequency.

\subsection{Energy consumption}

Table 2 shows the minimum and maximum energy consumption for the sender, the receiver and their combination. As expected, the energy consumption of the sender is always less than that of the receiver, since the receiver needs to write the file in NAND. The sender's minimum energy 
consumption occurs at different speed combinations (sender at $208 \mathrm{MHz}$, receiver at $520 \mathrm{MHz}$ ) than that of the receiver (sender at $208 \mathrm{MHz}$, receiver at $208 \mathrm{MHz}$ ). The minimum combined energy consumption however occurs when both the sender and the receiver operate at $208 \mathrm{MHz}$; the same speed combination that results in the minimum receiver energy consumption. In that operating point, the sender energy consumption increases by $2.1 \%$. However, if the network were to operate at the sender's optimal point, the receiver's energy consumption would rise by $45.7 \%$ and the combined energy consumption would increase by $23.4 \%$.

With the sender CPU at $104 \mathrm{MHz}$ and the receiver at 624 $\mathrm{MHz}$, the combined energy consumption is $67.3 \%$ higher than the minimum. At the lowest CPU speed, the sender cannot fill its transmit buffers fast enough; therefore the network bandwidth is not fully utilized, leading to an increase in latency. The wireless interface on both the sender and the receiver then needs to stay on for longer, therefore increasing the total energy consumption. At the same time, the receiver operates at its highest speed but, since the sender cannot transmit data fast enough, the receiver's CPU is idling (with a high idle power dissipation) for a considerable amount of time. With the sender at $624 \mathrm{MHz}$, the combined energy consumption is $60.8 \%$ higher than the minimum. Since the network transfer operation is not CPUbound for all but the lowest sender/receiver CPU speeds, the CPU is not fully utilized past a certain point, leading to increased energy consumption. Therefore, selecting appropriate CPU speeds for both the sender and receiver can drastically affect the energy consumption of a network transfer.

We note that it is not always possible to know the appropriate CPU speed at runtime a priori, using only static analysis. This is particularly the case for dynamic network operations, where the available network bandwidth can fluctuate due to congestion, loss and other factors. Therefore, in such situations, an on-line profiling tool such as Endoscope can provide immediate energy feedback to a CPU governor-or other resource and performance arbiter - and thereby discover the optimal operating point in a dynamic fashion.

Table 3 shows the minimum energy-latency product for the sender, receiver and their combination. The combined minimum energy-latency product occurs at the same CPU speed combination as the minimum combined energy consumption, unlike Sections 4.1 and 4.2. This is a highly desirable result, as it indicates that when both the sender and the receiver operate at $208 \mathrm{MHz}$, the energy consumption is not only minimized but is also optimally utilized to produce the best energy-latency tradeoff.

\subsection{Profiling Network Communication with Endoscope}

The introduction of the new LEAP2 and EMAP2 architecture and its Energy Endoscope is intended to enable a

\begin{tabular}{|c|cc|}
\hline & \multicolumn{2}{|c|}{ Min E.L.P (kJ*sec) } \\
& Value & $\begin{array}{c}\text { CPU Speed } \\
\text { (Snd/Rcv) }\end{array}$ \\
\hline Sender & 1.15 & 208624 \\
Receiver & 1.43 & 208208 \\
Combined & 2.68 & 208208 \\
\hline
\end{tabular}

Table 3. Minimum sender, receiver and combined energy - latency product and sender - receiver CPU speed for a 20 MB file transfer over $802.11 \mathrm{~b}$.

WSN system designer to accurately profile energy dissipation not previously visible. A direct demonstration of this is described below, for the fundamental example of data transport between two nodes, where large energy reduction is obtained in the optimized system without performance loss.

Figure 9 shows the power dissipation over time of the wireless, CPU, SDRAM and NAND subsystems, for the sender and the receiver, with both nodes' CPUs at their optimal energy consumption operating point of $208 \mathrm{MHz}$. The wireless interface draws the largest amount of power in both the sender and the receiver. Moreover, the difference in power dissipation of the two interfaces is negligible, as it is around $1 \mathrm{~mW}$. More importantly, however, the wireless power dissipation over time is constant, regardless of whether the interface is transmitting, receiving or idle. This is a well-studied property of $801.11 \mathrm{~b}$ and in sharp contrast with lower-power radios such as 802.15.4. In terms of energy-efficient system design, this result implies that significant energy gains can be achieved by keeping the wireless interface in powerdown state most of the time and only activate it when necessary $[13,1,14]$.

In terms of CPU power dissipation, there are some observable differences between the sender and the receiver. First, the receiver's CPU power dissipation is higher than the sender's and close to the maximum power dissipation $(279 \mathrm{~mW})$ for the $208 \mathrm{MHz}$ frequency, indicating a higher CPU utilization on the receiver. As previously noted, this is due to the fact that the receiver has to store the file in NAND flash which requires a significant amount of CPU time as well as SDRAM usage (Section 4.2).

In the first 7 seconds of the file transfer, the receiver's CPU power reaches its maximum value, while the sender's CPU is at its minimum value (excluding the time when the CPU is idle). At the same time, SDRAM power draw on the receiver is low and NAND power draw is high. This indicates that the YAFFS2 filesystem is executing block erases on NAND during that time, as shown in Section 4.2. During that time, writes cannot proceed at full speed, so the network bandwidth is not fully utilized; therefore the sender's CPU power dissipation is kept fairly low. After the erase operations complete on the receiver, the writes can proceed at 
full speed, the network can be fully utilized and the sender's CPU and SDRAM power dissipation rises.

SDRAM is used at the receiver for network buffer storage but more importantly, for write caching. The combination of those two operations results in considerable SDRAM power dissipation at the receiver-up to $50 \mathrm{~mW}$. NAND power dissipation over time at the receiver is similar to that of Figure 7. At the sender, NAND power draw is negligible.

\section{Related Work}

This section focuses on prior work most closely related to the energy endoscope in terms of hardware and software.

PASTA [16] was one of the first WSN platforms that targeted modularity and utilized high performance components. It included power monitoring capability and devicelevel power management. MPlatform [10] emphasizes a modular design and includes a custom inter-module bus protocol designed for high efficiency data transfers. Raghunathan et al. [15] studied subsystem power consumption on a modified Stargate-2 platform, however their work was focused on bench-top analysis. The previous LEAP [13] platform utilized a microcontroller architecture for power management functionality. With LEAP2, we augmented the measurement fidelity for detailed per-component energy usage data and lowered measurement overhead allowing for higher sampling rates over the previous LEAP design.

PowerTOSSIM [17] is a power-aware extension to the TOSSIM simulator. It uses an empirically-generated model of hardware behavior to simulate power dissipation on mote-class devices. The model is obtained by instrumenting and profiling a single node during actual operations. An oscilloscope was used to measure power dissipation of the entire mote. Since the oscilloscope does not provide persubsystem resolution as EMAP2 does, the authors used a set of microbenchmarks that exercised each subsystem independently. Even though subsystem independence can apply to a highly integrated, low-power system such as the mote, our experiments indicate that in a complex 32-bit node such as LEAP2, subsystem independence cannot be assumed.

Jung et al. [9] introduce a model-based design tool that can identify the dominant energy-intensive hardware components over a set of operating patterns. The authors propose several operating states where the system components are operating in different power modes. Measured power values can be used to populate the model parameters. The proposed models assume that power dissipation of subsystems is constant among operations. However, our experimental data suggests that this is not always the case, especially when a DVFS-enabled CPU is present.

SPOT [8] is an integrated add-on board that enables energy accounting on mote platforms. Charge accumulation is performed via a voltage to frequency converter circuit, sim-
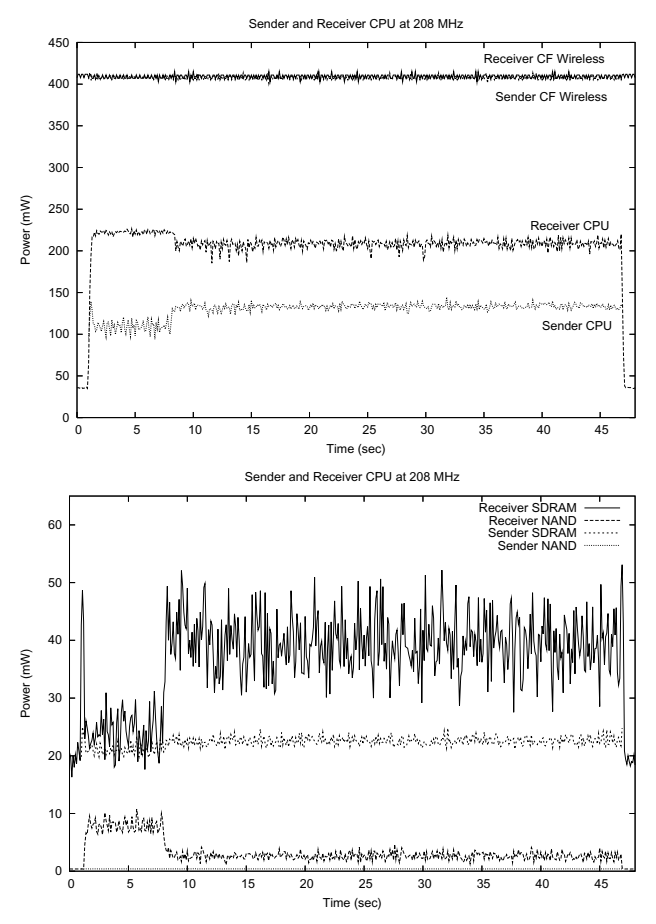

Figure 9. CPU, SDRAM, NAND and wireless power draw over time during a $20 \mathrm{MB}$ file transfer over $802.11 \mathrm{~b}$, with the sender and the receiver operating at $208 \mathrm{MHz}$.

ilar to a sigma-delta ADC architecture, achieving high resolution output and large dynamic range. The SPOT module also includes a dedicated counter value to provide charge accumulation timing information. Similar to the first generation LEAP, SPOT utilizes the I2C serial bus for the readback channel. We found the $\mathrm{I} 2 \mathrm{C}$ bandwidth limitation to hinder low overhead measurement at high sample rates and thus utilized a higher performance memory bus in LEAP2. Additionally, SPOT is a single-channel design, monitoring only the mote platform's input current and cannot easily support per-subsystem power information.

Triage [1] is a tiered hardware and software architecture for microservers. Similar to LEAP2, Triage uses a high-power platform with ample resources and a resourceconstrained low-power platform. The low-power platform schedules tasks to optimize the use of the high-power platform, based on profiling of execution time and energy usage. The profiler uses information about remaining battery energy as well as energy consumption during a particular operation. However, the profiler does not provide persubsystem information or power-tracing capabilities; instead, an external data acquisition board must be used.

Powerscope [5] combines hardware instrumentation and kernel software support to provide per-process energy usage information. Unlike etop, powerscope uses an external digi- 
tal multimeter and a second computer for data collection; in addition, data processing does not happen in real-time. Furthermore, the power-measuring system itself requires significant energy and physical resources to operate, thereby limiting its application in large-scale systems.

ECOsystem [18] incorporates energy as a "first-class resource" in OS scheduling, through the currentcy abstraction. Per-process energy information is critical in ECOsystem as processes need to be charged appropriately, to enable energy-based scheduling. ECOsystem uses a combination of battery lifetime sampling (a system-wide energy measurement) and system modelling. Our work is complimentary to ECOsystem since we focus on accurate and detailed real-time energy measurements as opposed to energy-aware operating system abstractions and modifications.

\section{Conclusion}

In this paper we presented the Energy Endoscope, a new hardware and software architecture that provides unprecedented visibility into the energy consumption of 32bit Wireless Sensor Nodes. We introduced the LEAP2 platform and its EMAP2 ASIC-based energy accounting unit that allows for low-power, integrated, real-time energy observation of individual platform components. In addition, we developed two software tools, etop and endoscope intended for both pre-deployment tradeoff analysis and postdeployment real-time profiling of several important subsystems such as CPU, memory, storage and networking. The new LEAP2 and EMAP2 ASIC and associated Energy Endoscope software systems have successfully met the challenge of providing real-time, high rate sampling at the hardware subsystem level and with very low overhead in terms of operating power, processor and memory utilization.

The Energy Endoscope system is now generally applicable to many WSN platforms. Experiments reported here demonstrate that the Endoscope can reveal the magnitude, temporal dependence and origin of energy dissipation in complex systems, where multiple forms of resource contention are present. As an example, we discovered significant differences in temporal dependence of power dissipation that exist between two different storage technologies, as well as the considerable effect of CPU power dissipation on storage and networking operations. By profiling subsystem energy during a network file transfer, we discovered the optimal CPU operating points for both the sender and receiver that can induce more than $60 \%$ energy savings without reduction in communication performance. These results demonstrate that a wide range of important research opportunities appear ahead for optimization of WSN systems.

\section{Acknowledgements}

We would like to thank the anonymous reviewers as well as our shepherd, Vijay Raghunathan, for their insightful comments, suggestions and feedback. This work was supported by NSF grants CNS-0453809 and ANI-0331481.

\section{References}

[1] N. Banerjee, J. Sorber, M. D. Corner, S. Rollins, and D. Ganesan. Triage: Balancing Energy Consumption and Quality of Service in a Microserver. In MobiSys '07.

[2] G. Banga, P. Druschel, and J. Mogul. Resource containers: A new facility for resource management in server systems. In $O S D I$ ' 99 .

[3] M. Desnoyers and M. R. Dagenais. The lttng tracer: A low impact performance and behavior monitor for gnu/linux. In Proceedings of Linux Symposium, Ottawa, Canada, 2006.

[4] Y. Diao, D. Ganesan, G. Mathur, and P. J. Shenoy. Rethinking data management for storage-centric sensor networks. In CIDR, pages 22-31, 2007.

[5] J. Flinn and M. Satyanarayanan. Powerscope: a tool for profiling the energy usage of mobile applications. In Second IEEE Workshop on Mobile Computing Systems and Applications, Feb. 1999.

[6] R. Gonzalez and M. Horowitz. Energy dissipation in general purpose microprocessors. In IEEE Journal of Solid-State Circuits, volume 31(9), pages 1277-1284, 1996.

[7] R. Jejurikar and R. Gupta. Dynamic voltage scaling for system-wide energy minimization in real-time embedded systems. In ISLPED '04.

[8] X. Jiang, P. Dutta, D. Culler, and I. Stoica. Micro power meter for energy monitoring of wireless sensor networks at scale. In IPSN '07.

[9] D. Jung, T. Teixeira, A. Barton-Sweeney, and A. Savvides. Model-based design exploration of wireless sensor node lifetimes. In EWSN 2007.

[10] D. Lymberopoulos, N. B. Priyantha, and F. Zhao. mplatform: a reconfigurable architecture and efficient data sharing mechanism for modular sensor nodes. In IPSN '07.

[11] D. Lymberopoulos and A. Savvides. Xyz: a motion-enabled, power aware sensor node platform for distributed sensor network applications. In IPSN ' 05 .

[12] G. Mathur, P. Desnoyers, D. Ganesan, and P. Shenoy. Ultralow power data storage for sensor networks. In IPSN '06.

[13] D. McIntire, K. H. Hing, B. Yip, A. Singh, W. Wu, and W. Kaiser. The low power energy aware processing (leap) system. In IPSN '06.

[14] T. Pering, V. Raghunathan, and R. Want. Exploiting radio hierarchies for power-efficient wireless discovery and connection setup. In 18th Intl. Conf. on VLSI Design, 2005.

[15] V. Raghunathan, T. Pering, R. Want, A. Nguyen, and P. Jensen. Experience with a low-power, wireless, mobile computing platform. In ISLPED '04.

[16] B. Schott, M. Bajura, J. Czarnaski, J. Flidr, T. Tho, and L. Wang. A modular power-aware microsensor with $1000 \mathrm{x}$ dynamic power range. In IPSN '05.

[17] V. Shnayder, M. Hempstead, B. Chen, G. W. Allen, and M. Welsh. Simulating the power consumption of large-scale sensor network applications. In SenSys '04.

[18] H. Zeng, X. Fan, C. Ellis, A. Lebeck, and A. Vahdat. ECOSystem: Managing energy as a first class operating system resource. In ASPLOS X, Oct 2002. 